Article

\title{
Galaxy Formation, Evolution and Rotation as a 4D Relativistic Cloud-World Embedded in a 4D Conformal Bulk
}

\author{
Mohammed B. Al-Fadhli 1,* \\ 1 College of Science, University of Lincoln, Lincoln, LN6 7TS, UK. \\ * Correspondence: malfadhli@lincoln.ac.uk; mo.fadhli7@gmail.com
}

\begin{abstract}
The recent observation of the G2 gas cloud orbit around the galactic centre has challenged the model of a mere supermassive black hole that should have destroyed it. In addition, the Planck Legacy 2018 (PL18) release has preferred a positively curved early Universe with a confidence level exceeding $99 \%$. In this study, the formation of a galaxy from the collapse of a supermassive gas cloud in the early Universe is modelled based on interaction field equations as a $4 \mathrm{D}$ relativistic cloud-world that flows and spins through a $4 \mathrm{D}$ conformal bulk of a primordial positive curvature considering the preference of the PL18 release. Owing to the curved background, this scenario of galaxy formation reveals that the core of the galaxy undergoes a forced vortex formation with a central event horizon leading to opposite vortices (traversable wormholes) that are spatially shrinking through evolving in the conformal time. It indicates that the galaxy and its core are formed at the same process where the surrounding gas clouds form the spiral arms due to the frame-dragging induced by the fastrotating core. Accordingly, the G2 gas cloud that only faced the drag effects could be explained if its orbit is around one of the traversable wormholes but at a distance from the central event horizon. Further, the simulation of the cloud-world flow through a positively curved early bulk demonstrates the fast orbital speed of outer stars owing to external fields exerted on galaxies as they have travelled through conformally curved spacetimes. These findings could explain the fast orbital speed of outer stars while the formation of a galaxy and its core simultaneously could explain the formation of the supermassive compact galaxy cores with a mass of $\sim 10^{9} M \odot$ at just $6 \%$ of the current Universe age.
\end{abstract}

Keywords: Galaxy Formation; Conformal Spacetime; Brane-World Modified Gravity.

\section{Introduction}

After the formulation of Einstein's general relativity utilizing 4D spacetime, Kaluza discovered in 1919 a potential field unification of gravitation and electromagnetism in 5D spacetime. Afterwards, Klein posited that the extra dimension could be compactified. However, those attempts and their expansions to more dimensions have not culminated in testable predictions nor competence to elucidate observations yet. An alternative to compactification, Gogberashvili, Randall and Sundrum in 1999 showed that the weak force of gravity can be explained using a model of $4 \mathrm{D}$ spacetime embedded in a negatively curved and large fifth dimension; nevertheless, it required massive gravitons [1-3].

Recently, the Planck Legacy 2018 (PL18) release has confirmed the existence of an enhanced lensing amplitude in the cosmic microwave background power spectra, which prefers a positively curved early Universe with a confidence level greater than $99 \%[4,5]$. Additionally, in 2021, the observation of the G2 gas cloud orbit around the galactic centre has challenged the model of a mere supermassive black hole at the centre of our galaxy that should have destroyed it $[6,7]$.

Considering the preferred primordial curvature by the PL18 release, this study aims for the field unification of gravitation and electromagnetism where celestial objects are considered as $4 \mathrm{D}$ relativistic cloud-worlds that flow and spin through a $4 \mathrm{D}$ conformal bulk of a primordial positive curvature. The derived interaction field equations are utilized to explain the recent observations of the G2 gas cloud orbit around the galactic centre. 


\section{Interaction Field Equations}

The PL18 release has preferred a positively curved early Universe, that is, is a sign of a primordial background curvature or a curved bulk. To incorporate the bulk curvature and its evolution over the conformal time, a modulus of spacetime deformation, $E_{D}$ in terms of energy density, is introduced based on the theory of elasticity [8]. The modulus can be expressed in terms of the resistance of the bulk to the localized curvature induced by celestial objects using Einstein field equations or in terms of the field strength of the bulk using the Lagrangian formulation of a non-interactive energy density existing in the bulk as a manifestation of the vacuum energy density as follows

$$
E_{D}=\frac{T_{\mu \nu}-T g_{\mu \nu} / 2}{R_{\mu \nu} / \mathcal{R}}=\frac{-\mathcal{F}_{\lambda \rho} \mathcal{F}^{\lambda \rho}}{4 \mu_{0}}
$$

where the stress is signified by the stress-energy tensor $T_{\mu \nu}$ of trace $T$ while the strain is signified by the Ricci curvature tensor $R_{\mu \nu}$ as the change in the curvature divided by the scalar of the bulk curvature $\mathcal{R}$ as the background or conformal curvature. $\mathcal{F}_{\lambda \rho}$ is the field strength tensor and $\mu_{0}$ is vacuum permeability. By incorporating the bulk influence, the Einstein-Hilbert action can be extended to

$$
S=E_{D} \int_{C}\left[\frac{R}{\mathcal{R}}+\frac{L}{\mathcal{L}}\right] \sqrt{-g} d^{4} \rho
$$

where $R$ is the Ricci scalar curvature representing the localized curvature induced into the bulk by a celestial object that is regarded as a 4 D relativistic cloud-world of metric $g_{u v}$ and Lagrangian density $L . \mathcal{R}$ represents the scalar curvature of the $4 \mathrm{D}$ bulk of metric $\tilde{g}_{\mu v}$ while $\mathcal{L}$ is the Lagrangian density of the bulk as a manifestation of its internal stresses and momenta reflecting its curvature. Since the bulk modulus, $E_{D}$, is constant with regards the cloud-world action under constant vacuum energy density condition and by considering the bulk expansion over the conformal time owing to the Universe expansion and its implication on the field strength of the bulk, a dual-action concerning the conservation of energy on global (bulk) and local (cloud-world) scales can be introduced as follows

$$
S=\int_{B}\left[\frac{-\mathcal{F}_{\lambda \rho} \tilde{g}^{\lambda \gamma} \mathcal{F}_{\gamma \alpha} \tilde{g}^{\rho \alpha}}{4 \mu_{0}}\right] \sqrt{-\tilde{g}} \int_{C}\left[\frac{R_{\mu \nu} g^{\mu \nu}}{\mathcal{R}_{\mu \nu} \tilde{g}^{\mu \nu}}+\frac{L_{\mu \nu} g^{\mu \nu}}{\mathcal{L}_{\mu \nu} \tilde{g}^{\mu \nu}}\right] \sqrt{-g} d^{4} \rho d^{4} \sigma
$$

where the relationship of the conformal bulk metric $\tilde{g}_{\mu \nu}$ with the embedded cloud-world metric $g_{u v}$ can be characterized by Weyl's conformal transformation as $\tilde{g}_{\mu \nu}=g_{\mu \nu} \Omega^{2}$, here $\Omega^{2}$ is a conformal function [9]. The global-local action should hold for any variation as

$$
\delta S=\int_{B}\left[\begin{array}{c}
\frac{-\delta\left(\mathcal{F}_{\lambda \rho} \tilde{g}^{\lambda \gamma} \mathcal{F}_{\gamma \alpha} \tilde{g}^{\rho \alpha}\right) \sqrt{-\tilde{g}}}{4 \mu_{0}} \\
-\frac{\mathcal{F}_{\lambda \rho} \tilde{g}^{\lambda \nu} \mathcal{F}_{\gamma \alpha} \tilde{g}^{\rho \alpha} \delta \sqrt{-\tilde{g}}}{4 \mu_{0}}
\end{array}\right] \int_{C}\left[\begin{array}{l}
\frac{\delta\left(R_{\mu \nu} g^{\mu \nu}\right) \sqrt{-g}}{\mathcal{R}_{\mu \nu} \tilde{g}^{\mu \nu}}-\frac{\delta\left(\mathcal{R}_{\mu \nu} \tilde{g}^{\mu \nu}\right) R_{\mu \nu} g^{\mu \nu} \sqrt{-g}}{\left(\mathcal{R}_{\mu \nu} \tilde{g}^{\mu \nu}\right)^{2}}+\frac{R_{\mu \nu} g^{\mu \nu} \delta \sqrt{-g}}{\mathcal{R}_{\mu \nu} \tilde{g}^{\mu \nu}} \\
+\frac{\delta\left(L_{\mu \nu} g^{\mu \nu}\right) \sqrt{-g}}{\mathcal{L}_{\mu \nu} \tilde{g}^{\mu \nu}}-\frac{\delta\left(\mathcal{L}_{\mu \nu} \tilde{g}^{\mu \nu}\right) L_{\mu \nu} g^{\mu \nu} \sqrt{-g}}{\left(\mathcal{L}_{\mu \nu} \tilde{g}^{\mu \nu}\right)^{2}}+\frac{L_{\mu \nu} g^{\mu \nu} \delta \sqrt{-g}}{\mathcal{L}_{\mu \nu} \tilde{g}^{\mu \nu}}
\end{array}\right] d^{4} \rho d^{4} \sigma
$$

By utilizing Jacobi's formula, $\delta \sqrt{-g}=-\sqrt{-g} g_{\mu \nu} \delta g^{\mu \nu} / 2$ [10]. Hence, the variation is

$$
\delta S=\int_{B}\left[\begin{array}{c}
-\frac{\mathcal{F}_{\lambda \rho} \mathcal{F}_{\gamma}^{\rho} \delta \tilde{g}^{\lambda \gamma}+\delta \mathcal{F}_{\lambda \rho} \mathcal{F}^{\lambda \rho}}{2 \mu_{0}} \\
+\mathcal{F}_{\lambda \rho} \tilde{g}^{\lambda \gamma} \mathcal{F}_{\gamma \alpha} \tilde{g}^{\rho \alpha} \frac{\tilde{g}_{\mu \nu} \delta \tilde{g}^{\mu \nu}}{8 \mu_{0}}
\end{array}\right] \sqrt{-\tilde{g}} \int_{C}\left[\begin{array}{c}
\frac{R_{\mu \nu} \delta g^{\mu \nu}+g^{\mu \nu} \delta R_{\mu \nu}}{\mathcal{R}}-\frac{\mathcal{R}_{\mu \nu} \delta \tilde{g}^{\mu \nu}+\tilde{g}^{\mu v} \delta \mathcal{R}_{\mu \nu}}{\mathcal{R}^{2}} R-\frac{g_{\mu \nu} \delta g^{\mu \nu}}{2 \mathcal{R}} R \\
+\frac{L_{\mu \nu} \delta g^{\mu \nu}+g^{\mu \nu} \delta L_{\mu \nu}}{\mathcal{L}}-\frac{\mathcal{L}_{\mu \nu} \delta \tilde{g}^{\mu \nu}+\tilde{g}^{\mu \nu} \delta \mathcal{L}_{\mu \nu}}{\mathcal{L}^{2}} L-\frac{g_{\mu \nu} \delta g^{\mu \nu}}{2 \mathcal{L}} L
\end{array}\right] \sqrt{-g} d^{4} \rho d^{4} \sigma(5)
$$

where the electromagnetic and Lagrangian densities are considered on the boundaries. 
By considering the cloud-world's boundary term: $\int_{c} g^{\mu v} \delta R_{\mu \nu} \sqrt{-g} d^{4} \sigma / \mathcal{R}$, the variation in the Ricci curvature tensor $\delta R_{u v}$ can be expressed in terms of the covariant derivative of the difference between two Levi-Civita connections, the Palatini identity: $\delta R_{\mu \nu}=$ $\nabla_{\rho}\left(\delta \Gamma_{v \mu}^{\rho}\right)-\nabla_{v}\left(\delta \Gamma_{\rho \mu}^{\rho}\right)$, where the variation with respect to the inverse metric $g^{\mu \nu}$ can be obtained by using the metric compatibility of the covariant derivative, $\nabla_{\rho} g^{\mu \nu}=0$ [10], as $g^{\mu \nu} \delta R_{\mu \nu}=\nabla_{\rho}\left(g^{\mu \nu} \delta \Gamma_{v \mu}^{\rho}-g^{\mu \rho} \delta \Gamma_{\sigma \mu}^{\sigma}\right)$. Consequently, the cloud-world's boundary term as a total derivative for any tensor density can be transformed based on Stokes' theorem with renaming the dummy indices as follows

$$
\begin{gathered}
\int_{C}\left[\frac{g^{\mu \nu} \delta R_{\mu \nu}}{\mathcal{R}}\right] \sqrt{-g} d^{4} \rho=\frac{1}{\mathcal{R}} \int_{C}\left[\nabla_{\rho}\left(g^{\mu \nu} \delta \Gamma_{\nu \mu}^{\rho}-g^{\mu \rho} \delta \Gamma_{\sigma \mu}^{\sigma}\right)\right] \sqrt{-g} d^{4} \rho \\
=\frac{1}{\mathcal{R}} \int_{C}\left[\nabla_{\mu} H^{\mu}\right] \sqrt{-g} d^{4} \rho=\frac{\epsilon}{\mathcal{R}} \int_{\partial C}[K] \sqrt{|q|} d^{3} \varrho
\end{gathered}
$$

where the bulk scalar curvature, $\mathcal{R}$, is left outside the integral transformation as it only acts as a scalar to the transformation. The same transformations are applied to the bulk and Lagrangian boundary terms and the electromagnetic tensor variation as it represents a boundary term. Thus, the transformed boundary action, $S_{b}$, along with a transformed global boundary term are

$$
S_{b}=\int_{\partial B} \epsilon\left[\frac{J \rho}{2}\right] \sqrt{-\tilde{q}} d^{3} \varsigma\left(\begin{array}{l}
\frac{\epsilon}{\mathcal{R}} \int_{\partial C}[K] \sqrt{|q|} d^{3} \varrho-\frac{R \epsilon}{\mathcal{R}^{2}} \int_{\partial C}[\mathcal{K}] \sqrt{|q|} d^{3} \varrho \\
+\frac{\epsilon}{\mathcal{L}} \int_{\partial C}[l] \sqrt{|q|} d^{3} \varrho-\frac{L \epsilon}{\mathcal{L}^{2}} \int_{\partial C}[\ell] \sqrt{|q|} d^{3} \varrho
\end{array}\right)
$$

where $K$ and $\mathcal{K}$ are the traces of the cloud-world and the bulk extrinsic curvatures, $l$ and $\ell$ are the extrinsic traces of the Lagrangian density on the cloud-world and the bulk boundaries, $q$ and $q$ are the determinants of their induced metrics respectively, and $\epsilon$ equals 1 when the normal $\hat{n}_{u}$ is a spacelike entity and equals -1 when it is a timelike entity. $J_{\rho}$ is the four current. The boundary action should hold for any variation and by considering the transformed cloud-world's boundary term, the variation in the term yields

$$
\frac{\epsilon}{\mathcal{R}} \int_{\partial C}\left[K_{\mu \nu} \delta q^{\mu \nu}+q^{\mu \nu} \delta K_{\mu \nu}+K \frac{\delta \sqrt{|q|}}{\sqrt{|q|}}\right] \sqrt{|q|} d^{3} \varrho
$$

where $K=K_{\mu \nu} q^{\mu \nu}$. By utilising Jacobi's formula for the determinant differentiation; thus, $\delta \sqrt{|q|}=-\sqrt{|q|} q_{\mu \nu} \delta q^{\mu \nu} / 2$ and by utilising the variation in the metric times the inverse metric, $q^{\mu \nu} q_{\mu \nu}=\delta_{v}^{\mu}$ as $q^{\mu \nu}=-q_{\mu \nu} \delta q^{\mu \nu} / \delta q_{\mu \nu}$; thus, the boundary term is

$$
\frac{\epsilon}{\mathcal{R}} \int_{\partial C}\left[K_{\mu \nu} \delta q^{\mu \nu}-\frac{1}{2} K\left(q_{\mu \nu} \delta q^{\mu \nu}+2 q_{\mu \nu} \frac{\delta K_{\mu \nu}}{\delta q_{\mu \nu} K} \delta q^{\mu \nu}\right)\right] \sqrt{|q|} d^{3} \varrho
$$

here $\delta K_{\mu \nu} / \delta q_{\mu \nu} K=\left(\delta K_{\mu \nu} / K_{\mu \nu}\right)\left(q_{\mu \nu} / \delta q_{\mu \nu}\right)=\delta \ln K_{\mu \nu} / \delta \ln q_{\mu \nu}$ resembles the Ricci flow in a normalized form reflecting the conformal distortion in the boundary over the conformal time, which can be expressed as a positive function $\Omega^{2}$ according to Weyl's conformal transformation as $\tilde{q}_{\mu \nu}=q_{\mu \nu} \Omega^{2}$ [11]. Thus, the boundary term is

$$
\frac{\epsilon}{\mathcal{R}} \int_{\partial C}\left[K_{\mu \nu} \delta q^{\mu \nu}-\frac{1}{2} K \hat{q}_{\mu \nu} \delta q^{\mu \nu}\right] \sqrt{|q|} d^{3} \varrho
$$

where $\hat{q}_{\mu \nu}=q_{\mu \nu}+2 \tilde{q}_{\mu \nu}$ denoting the conformally transformed induced metric on the cloud-world boundary. The same is applied for the bulk and Lagrangian boundary terms. 
Thus, the variation in the whole action with renaming the dummy indices is

$$
\delta S=\left(\begin{array}{l}
-\int_{B}\left[\frac{1}{2 \mu_{0}}\left(\mathcal{F}_{\mu \lambda} \mathcal{F}_{v}^{\lambda}-\frac{\mathcal{F}_{\lambda \rho} \mathcal{F}^{\lambda \rho}}{4} \tilde{g}_{\mu \nu}\right)\right] \delta \tilde{g}^{\mu \nu} \sqrt{-\tilde{g}} d^{4} \sigma \\
-\int_{\partial B}\left[\frac{\epsilon}{2} \delta J_{v} / \delta \tilde{q}^{\mu \nu}\right] \delta \tilde{q}^{\mu \nu} \sqrt{|\tilde{q}|} d^{3} \varsigma
\end{array}\right)\left(\begin{array}{l}
\int_{C}\left[\frac{R_{\mu \nu}}{\mathcal{R}}-\frac{\mathcal{R}_{\mu v}}{\mathcal{R}^{2}} R-\frac{R}{2 \mathcal{R}} g_{\mu \nu}\right] \delta g^{\mu \nu} \sqrt{-g} d^{4} \rho \\
+\int_{C}\left[\frac{L_{\mu \nu}}{\mathcal{L}}-\frac{\mathcal{L}_{\mu v}}{\mathcal{L}^{2}} L-\frac{L}{2 \mathcal{L}} g_{\mu \nu}\right] \delta g^{\mu \nu} \sqrt{-g} d^{4} \rho \\
+\int_{\partial C}\left[\frac{\epsilon}{\mathcal{R}}\left(K_{\mu \nu}-\frac{1}{2} K \hat{q}_{\mu \nu}\right)\right] \delta q^{\mu \nu} \sqrt{|q|} d^{3} \varrho \\
-\int_{\partial C}\left[\frac{R \epsilon}{\mathcal{R}^{2}}\left(\mathcal{K}_{\mu \nu}-\frac{1}{2} \mathcal{K} \hat{q}_{\mu \nu}\right)\right] \delta q^{\mu \nu} \sqrt{|q|} d^{3} \varrho \\
+\int_{\partial C}\left[\frac{\epsilon}{\mathcal{L}}\left(l_{\mu \nu}-\frac{1}{2} l \hat{q}_{\mu \nu}\right)\right] \delta q^{\mu \nu} \sqrt{|q|} d^{3} \varrho \\
-\int_{\partial C}\left[\frac{L \epsilon}{\mathcal{L}^{2}}\left(\ell_{\mu \nu}-\frac{1}{2} \ell \hat{q}_{\mu \nu}\right)\right] \delta q^{\mu \nu} \sqrt{|q|} d^{3} \varrho
\end{array}\right)
$$

By encapsulating the new Lagrangian terms on boundaries into an extended stress-energy tensor defined as $\hat{T}_{\mu \nu}:=\left(2 L_{\mu \nu}-L \hat{g}_{\mu \nu}\right)-\left(2 l_{\mu \nu}-l \hat{q}_{\mu \nu}\right)+\left(2 \ell_{\mu \nu}-\ell \hat{q}_{\mu \nu}\right) L / \mathcal{L}$ that counts for the energy density and flux of the cloud-world, $L_{\mu \nu}$, and electromagnetic energy flux from its boundary, $l_{\mu \nu}$, over the conformal time where $\hat{g}_{\mu \nu}=g_{\mu \nu}+2 \tilde{g}_{\mu \nu}$ is the conformally transformed metric since $\tilde{g}_{\mu \nu}=\mathcal{L}_{\mu \nu} / \mathcal{L}=\mathcal{L}_{\mu \nu} / \mathcal{L}_{\mu \nu} \tilde{g}^{\mu \nu}$ while the Lagrangian density on bulk's boundary, $\ell_{\mu \nu}$, has $L / \mathcal{L}$ factor, i.e. it is only significant when the cloud-world has a high Lagrangian density such as black holes where the entire contribution belongs to the boundary terms when finding the black hole entropy $[12,13]$. The outcomes of the global action resembled an extended electromagnetic stress-energy tensor as $\mathcal{T}_{\mu \nu}:=\left(\mathcal{F}_{\mu \lambda} \mathcal{F}_{\nu}^{\lambda}-\right.$ $\left.\left.\mathcal{F}_{\lambda \rho} \mathcal{F}^{\lambda \rho} \tilde{g}_{\mu \nu} / 4\right) / \mu_{0}\right)+\delta J_{v} / \delta \tilde{q}^{\mu \nu}$ denoting energy density exists in the bulk as the vacuum energy density in addition to the variation of the four current with regards bulk's boundary variation over conformal time. From Equations (1), (2) and (11), $\mathcal{T}_{\mu \nu}:=E_{D}=\mathcal{L}=\mathcal{R} c^{4} / 8 \pi G_{t}$ is proportional to the fourth power of the speed of light that in turn is directly proportional to the frequency, which can be in accordance with frequency cut-off predictions of vacuum energy density in the quantum field theory $[14,15]$. By choosing $\epsilon$ as a timelike entity and applying the principle of stationary action yields

$$
\frac{R_{\mu \nu}}{\mathcal{R}}-\frac{1}{2} \frac{R}{\mathcal{R}} g_{\mu \nu}-\frac{\mathcal{R}_{\mu \nu}}{\mathcal{R}^{2}} R+\frac{R\left(\mathcal{K}_{\mu \nu}-\frac{1}{2} \mathcal{K} \hat{q}_{\mu \nu}\right)-\mathcal{R}\left(K_{\mu \nu}-\frac{1}{2} K \hat{q}_{\mu \nu}\right)}{\mathcal{R}^{2}}=\frac{\widehat{T}_{\mu \nu}}{\mathcal{T}_{\mu \nu}}
$$

The interaction field equations can be interpreted as indicating that the cloud-world's induced curvature over the bulk's pre-existing curvature equals the ratio of the cloudworld's imposed energy density and its flux to the bulk's vacuum energy density and its flux through the expanding/contracting Universe. By utilizing Equations (1) and (11) that state $\mathcal{J}_{\mu \nu}:=E_{D}=\mathcal{R} c^{4} / 8 \pi G_{t}$, the field equations can be simplified to

$$
R_{\mu \nu}-\frac{1}{2} R \hat{g}_{\mu \nu}+\frac{R\left(\mathcal{K}_{\mu \nu}-\frac{1}{2} \mathcal{K} \hat{q}_{\mu \nu}\right)-\mathcal{R}\left(K_{\mu \nu}-\frac{1}{2} K \hat{q}_{\mu \nu}\right)}{\mathcal{R}}=\frac{8 \pi G_{t}}{c^{4}} \widehat{T}_{\mu \nu}
$$

where $\hat{g}_{\mu \nu}=g_{\mu \nu}+2 \tilde{g}_{\mu \nu}$ is the conformally transformed metric counting for both cloudworld, $g_{\mu \nu}$, and bulk, $\tilde{g}_{\mu \nu}=\mathcal{R}_{\mu \nu} / \mathcal{R}=\mathcal{R}_{\mu \nu} / \mathcal{R}_{\mu \nu} \tilde{g}^{\mu \nu}$, metrics whereas Einstein spaces are subclass of conformal spaces [9]. The evolution in $G_{t}$ reflects the field strength evolution with Universe expansion and it can accommodate the bulk curvature evolution over the conformal time against constant $G$ for a special flat spacetime case. The new boundary term is only significant at high-energy limits such as within black holes [12]. These field equations could remove the singularities and satisfy a conformal invariance theory. 


\section{Galaxy Formation, Evolution and Rotation}

The entire contribution comes from the boundary term when calculating the black hole entropy using the semiclassical approach $[12,13]$. Applying this concept and by rearranging the field equations for this setting as

$$
\frac{R_{\mu \nu}}{\mathcal{R}}-\frac{1}{2} \frac{R}{\mathcal{R}} g_{\mu \nu}-\frac{\mathcal{R}_{\mu \nu}}{\mathcal{R}^{2}} R=\frac{\widehat{T}_{\mu \nu}}{\mathcal{T}_{\mu \nu}}-\frac{R\left(\mathcal{K}_{\mu \nu}-\frac{1}{2} \mathcal{K} \hat{q}_{\mu \nu}\right)-\mathcal{R}\left(K_{\mu \nu}-\frac{1}{2} K \hat{q}_{\mu \nu}\right)}{\mathcal{R}^{2}}=0
$$

The field equations can describe the interaction between a $4 \mathrm{D}$ relativistic cloud-world of intrinsic $R_{\mu \nu}$ and extrinsic $K_{\mu \nu}$ curvatures with a stress-energy $\widehat{T}_{\mu \nu}$ and the $4 \mathrm{D}$ bulk of intrinsic $\mathcal{R}_{\mu \nu}$ and extrinsic $\mathcal{K}_{\mu \nu}$ curvatures with a stress-energy $\mathcal{T}_{\mu \nu}$. From Equation (14), the field equations yield

$$
R_{\mu \nu}=\frac{1}{2} R g_{\mu \nu}+\frac{\mathcal{R}_{\mu \nu}}{\mathcal{R}} R=\frac{1}{2} R \hat{g}_{\mu \nu}=\frac{1}{2} R\left(g_{\mu \nu}+2 \widetilde{g}_{\mu \nu}\right)=\frac{1}{2} R g_{\mu \nu}\left(1+2 \Omega^{2}\right)=0
$$

where $\hat{g}_{\mu \nu}=g_{\mu \nu}+2 \tilde{g}_{\mu \nu}$ and $\tilde{g}_{\mu \nu}=\mathcal{R}_{\mu \nu} / \mathcal{R}=\mathcal{R}_{\mu \nu} / \mathcal{R}_{\mu \nu} \tilde{g}^{\mu \nu}$ is the conformal bulk metric, which can be expressed as proportional to cloud-world metric $g_{\mu \nu}$ as $\tilde{g}_{\mu \nu}=g_{\mu \nu} \Omega^{2}$ by utilizing $\Omega^{2}$, the conformal transformation function. The conformally transformed metric $\hat{g}_{\mu \nu}=g_{\mu \nu}\left(1+2 \Omega^{2}\right)$ can be expressed as

$d s^{2}=-A(r)\left(1+2 \Omega^{2}(r)\right) c^{2} d t^{2}+S^{2}\left(B(r)\left(1+2 \Omega^{2}(r)\right) d r^{2}+r^{2} d \theta^{2}+r^{2} \sin ^{2} \theta d \phi^{2}\right)$

where $A, B$ and $\Omega^{2}$ are functions of the radius $r$, and $S$ is a dimensionless conformal scale factor. By performing coordinate transformation as follows

$$
d s^{2}=-\left(A(\lambda)+2 A(\lambda) \Omega^{2}(\lambda)\right) c^{2} d t^{2}+\left(\left(B(\lambda)+2 B(\lambda) \Omega^{2}(\lambda)\right) d \lambda^{2}+\lambda^{2} d \theta^{2}+\lambda^{2} \sin ^{2} \theta d \phi^{2}\right)
$$

The Christoffel symbols of this metric are

$$
\begin{gathered}
\Gamma_{00}^{1}=\frac{\dot{A}\left(1+2 \Omega^{2}\right)+4 A \dot{\Omega}}{2\left(B+2 B \Omega^{2}\right)}, \quad \Gamma_{01}^{0}=\frac{\dot{A}\left(1+2 \Omega^{2}\right)+4 A \dot{\Omega}}{2\left(A+2 A \Omega^{2}\right)}, \quad \Gamma_{11}^{1}=\frac{\dot{B}\left(1+2 \Omega^{2}\right)+4 B \dot{\Omega}}{2\left(B+2 B \Omega^{2}\right)} \\
\Gamma_{22}^{1}=\frac{-\lambda}{\left(B+2 B \Omega^{2}\right)}, \quad \Gamma_{33}^{1}=\frac{-\lambda \sin ^{2} \theta}{\left(B+2 B \Omega^{2}\right)}, \quad \Gamma_{21}^{2}=\Gamma_{12}^{2}=\frac{1}{\lambda} \\
\Gamma_{33}^{2}=-\sin \theta \cos \theta, \quad \Gamma_{32}^{3}=\Gamma_{23}^{3}=\frac{\cos \theta}{\sin \theta}
\end{gathered}
$$

The Ricci tensor components are

$$
\begin{aligned}
R_{00}=-\frac{\frac{d}{d \lambda}\left(\dot{A}\left(1+2 \Omega^{2}\right)+4 A \dot{\Omega}\right)}{2\left(B+2 B \Omega^{2}\right)}-\frac{\dot{A}\left(1+2 \Omega^{2}\right)+4 A \dot{\Omega}}{2\left(B+2 B \Omega^{2}\right)} \frac{\dot{B}\left(1+2 \Omega^{2}\right)+4 B \dot{\Omega}}{2\left(B+2 B \Omega^{2}\right)} \\
+\frac{\dot{A}\left(1+2 \Omega^{2}\right)+4 A \dot{\Omega}}{2\left(B+2 B \Omega^{2}\right)} \frac{\dot{A}\left(1+2 \Omega^{2}\right)+4 A \dot{\Omega}}{2\left(A+2 A \Omega^{2}\right)}-\frac{1}{\lambda} \frac{\dot{A}\left(1+2 \Omega^{2}\right)+4 A \dot{\Omega}}{2\left(B+2 B \Omega^{2}\right)}
\end{aligned}
$$




$$
\begin{gathered}
R_{00}=-\frac{\ddot{A}\left(1+2 \Omega^{2}+4 \dot{\Omega}\right)+4 A \ddot{\Omega}+4 \dot{\Omega} \dot{A}}{2\left(B+2 B \Omega^{2}\right)}+\frac{\left(\dot{A}\left(1+2 \Omega^{2}\right)+4 A \dot{\Omega}\right)\left(\dot{B}\left(1+2 \Omega^{2}\right)+4 B \dot{\Omega}\right)}{4\left(B+2 B \Omega^{2}\right)^{2}} \\
+\frac{\left(\dot{A}\left(1+2 \Omega^{2}\right)+4 A \dot{\Omega}\right)^{2}}{4\left(A+2 A \Omega^{2}\right)\left(B+2 B \Omega^{2}\right)}-\frac{1}{\lambda} \frac{\dot{A}\left(1+2 \Omega^{2}\right)+4 A \dot{\Omega}}{\left(B+2 B \Omega^{2}\right)} \\
R_{11}=\frac{\frac{d}{d \lambda}\left(\dot{A}\left(1+2 \Omega^{2}\right)+4 A \dot{\Omega}\right)}{2\left(A+2 A \Omega^{2}\right)}+\frac{\left(\dot{A}\left(1+2 \Omega^{2}\right)+4 A \dot{\Omega}\right)^{2}}{4\left(A+2 A \Omega^{2}\right)^{2}}+\frac{1}{\lambda} \frac{\dot{B}\left(1+2 \Omega^{2}\right)+4 B \dot{\Omega}}{\left(B+2 B \Omega^{2}\right)} \\
+\frac{\left(\dot{A}\left(1+2 \Omega^{2}\right)+4 A \dot{\Omega}\right)\left(\dot{B}\left(1+2 \Omega^{2}\right)+4 B \dot{\Omega}\right)}{4\left(A+2 A \Omega^{2}\right)\left(B+2 B \Omega^{2}\right)} \\
R_{11}=\frac{1}{2}\left(\frac{\ddot{A}\left(1+2 \Omega^{2}+4 \dot{\Omega}\right)+4 A \ddot{\Omega}+4 \dot{\Omega} \dot{A}}{\left(A+2 A \Omega^{2}\right)}-\frac{\left(\dot{A}\left(1+2 \Omega^{2}\right)+4 A \dot{\Omega}\right)^{2}}{2\left(A+2 A \Omega^{2}\right)^{2}}\right) \\
-\frac{\left(\dot{A}\left(1+2 \Omega^{2}\right)+4 A \dot{\Omega}\right)\left(\dot{B}\left(1+2 \Omega^{2}\right)+4 B \dot{\Omega}\right)}{4\left(A+2 A \Omega^{2}\right)\left(B+2 B \Omega^{2}\right)}-\frac{1}{\lambda} \frac{\dot{B}\left(1+2 \Omega^{2}\right)+4 B \dot{\Omega}}{B+2 B \Omega^{2}} \\
R_{33}=\frac{\sin ^{2} \theta}{\left(B+2 B \Omega^{2}\right)}-\frac{\lambda \sin \theta}{2\left(B+2 B \Omega^{2}\right)}\left(\frac{\dot{B}\left(1+2 \Omega^{2}\right)+4 B \dot{\Omega}}{\left(B+2 B \Omega^{2}\right)}-\frac{\dot{A}\left(1+2 \Omega^{2}\right)+4 A \dot{\Omega}}{\left(A+2 A \Omega^{2}\right)}\right)-\sin ^{2} \theta \\
\left.R_{22}=\frac{1}{\left(B+2 B \Omega^{2}\right)}-\frac{\dot{B}\left(1+2 \Omega^{2}\right)+4 B \dot{\Omega}}{2\left(B+2 B \Omega^{2}\right)}-\frac{\dot{A}\left(1+2 \Omega^{2}\right)+4 A \dot{\Omega}}{\left(B+2 A \Omega^{2}\right)}\right)-1
\end{gathered}
$$

Using Ricci tensor components gives

$$
\left(\dot{A}\left(1+2 \Omega^{2}\right)+4 A \dot{\Omega}\right)\left(B+2 B \Omega^{2}\right)+\left(A+2 A \Omega^{2}\right)\left(\dot{B}\left(1+2 \Omega^{2}\right)+4 B \dot{\Omega}\right)=0
$$

Equation (25) leads to

$$
B+2 B \Omega^{2}=\frac{c}{A+2 A \Omega^{2}}
$$

where $c$ is a constant. The metric should approach the Minkowski metric at $\lambda \rightarrow \infty$; accordingly:

$$
\lim _{\lambda \rightarrow \infty}\left(A(\lambda)+2 A(\lambda) \Omega^{2}(\lambda)\right)=1
$$

Applying the weak-field limit:

$$
-A(\lambda)\left(1+2 \Omega^{2}(\lambda)\right)=-\left(1+\frac{2 \varphi}{c^{2}}\right)
$$

where $\varphi=-G M / \lambda$. 
By combining Equations (26) - (28) yields

$$
\Omega^{2}(\lambda)=-\frac{G M}{\lambda c^{2}}, \quad A(\lambda)=1, \quad B(\lambda)=\left(1-2 \frac{G M}{\lambda c^{2}}\right)^{-2}
$$

Equation (29) shows the conformal function $\Omega^{2}(\lambda)$ has a minus sign, revealing a spatial shrinking through evolving in the conformal time. This agrees with the vortex model which can occur due to the high-speed spinning. By performing the retransformation of the coordinates and then substituting Equation (29) to Equation (16), The conformally transformed metric $\hat{g}_{\mu \nu}=g_{\mu \nu}+2 \tilde{g}_{\mu \nu}=g_{\mu \nu}\left(1+2 \Omega^{2}\right)$ is

$$
d s^{2}=\left(1+2 \Omega^{2}\right)\left(-c^{2} d t^{2}+S^{2}\left(\frac{d r^{2}}{\left(1+2 \Omega^{2}\right)^{2}}+\frac{r^{2} d \theta^{2}+r^{2} \sin ^{2} \theta d \phi^{2}}{\left(1+2 \Omega^{2}\right)}\right)\right)
$$

where $S^{2}$ is a dimensionless spatial scale factor that accounts for the conformal shrinking. The denominator of the radial term can be interpreted as an intrinsic curvature indicator, where based on this, the metric on the two-sphere is warped by the conformal function $\Omega^{2}$. This metric is equivalent to the Schwarzschild metric and it could provide an interpretation to its terms. The metric can be visualized by using Flamm's paraboloid with considering the additional dimension as the conformal time as follows

$$
\eta(r)=\mp 2 \sqrt{\left(1+2 \Omega^{2}\right) r_{s} r}+k
$$

where $r_{s}$ is the Schwarzschild radius and $k$ is a constant. By choosing an appropriate $\mathrm{k}$ in Equation (31) for both positive and negative solutions, Figure 1 shows that the proper distances and their corresponding proper areas are increasing as they are evolving in the conformal time while the radius decreases

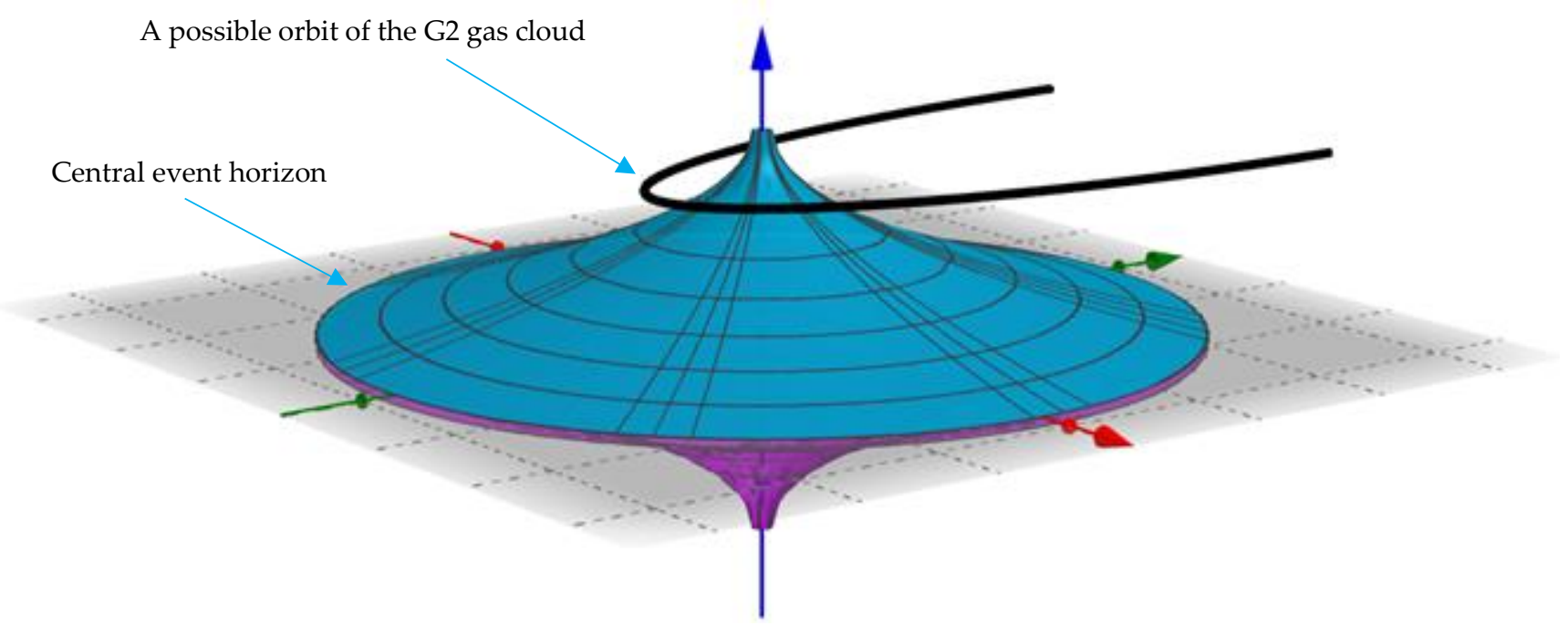

Figure 1. Evolution of the proper distances and their corresponding proper areas over the conformal time with the evolution of the radius. A possible orbit of the G2 gas cloud around the wormhole is suggested.

The increase in the proper distances and their corresponding proper areas as the radius decreases is also agreed with the vortex model as shown in Figure $2 b$ while the positive and negative solutions of Equation (31) can indicate the evolution of the vortex in opposite directions, i.e., forming a dual vortex perpendicular on the galaxy plane. 


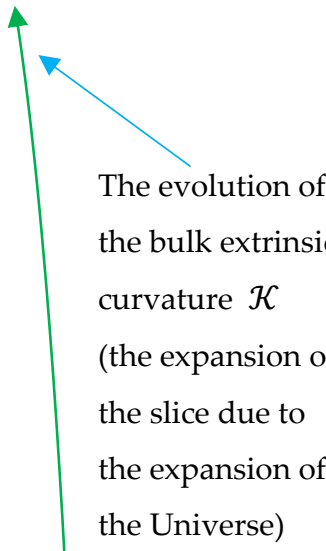

Figure 2 shows the evolution of the $4 \mathrm{D}$ cloud-world through its travel and spin in the conformal space-time of the $4 \mathrm{D}$ bulk.

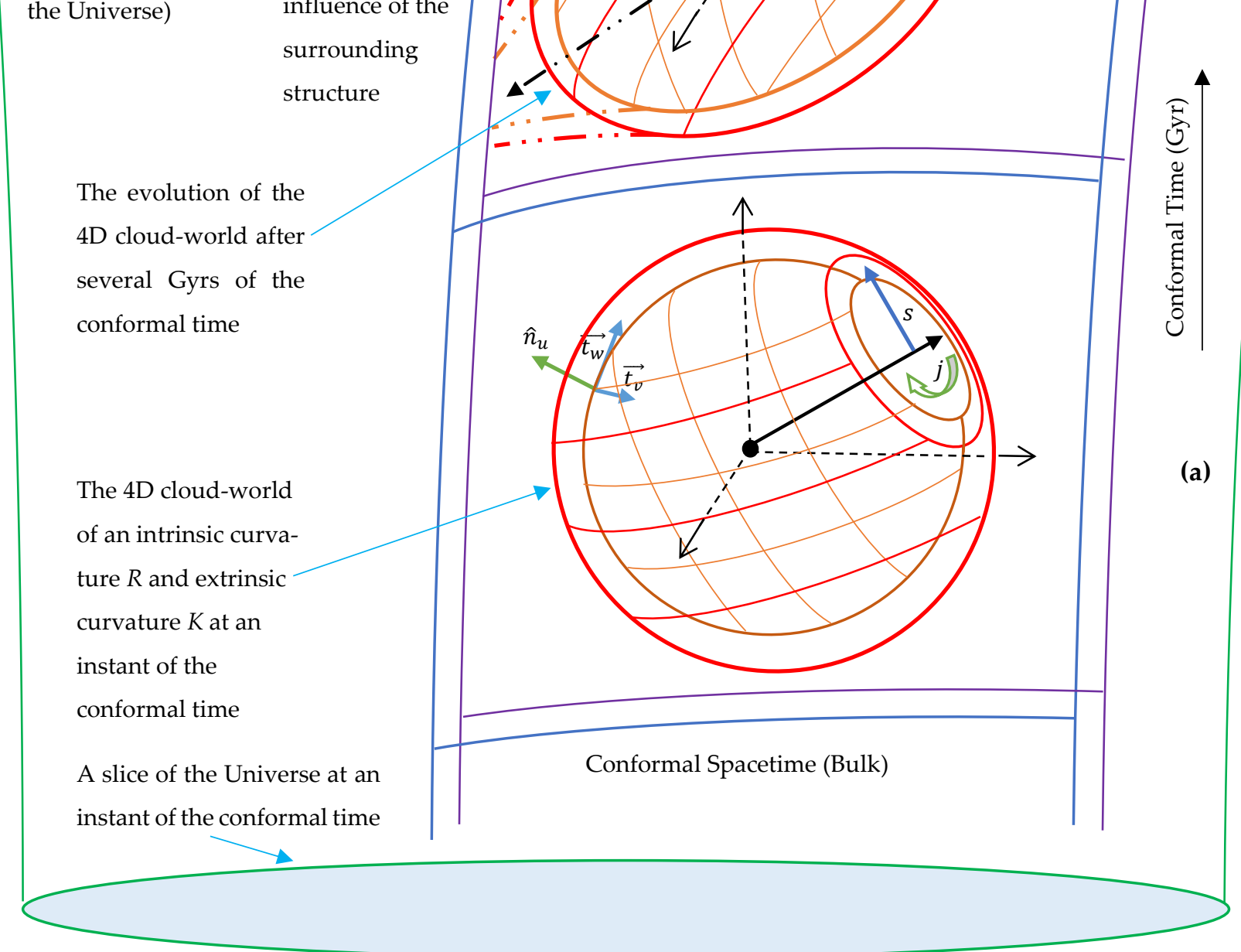

Figure 2. The hypersphere of a compact core of a galaxy (the red-orange $4 \mathrm{D}$ cloud-world) along with its travel and spin through the conformal spacetime (the blue-purple $4 \mathrm{D}$ bulk representing the background of distinctive curvature evolving over the conformal time.

The G2 cloud which only faced drag forces and was not destroyed [6,7]; therefore, orbiting a wormholes could explain these observations. In addition, the observations of the superluminal motion in the x-ray jet of M87 [16] could be travel through these traversable wormholes. 
To evaluate the influence of the spinning and the curvature of the background on the core of the galaxy and the surrounding gas clouds (the spiral arms), a fluid simulation was performed based on Newtonian dynamics by using the Fluid Pressure and Flow software [17]. In this simulation, the fluid was deemed to represent the spacetime continuum throughout incrementally flattening curvature paths representing conformal curvature evolution to analyze the external momenta exerted on objects flowing throughout the incrementally flattening curvatures. The momenta yielded by the fluid simulation were used to simulate a spiral galaxy as a forced vortex as shown in Figure 3.

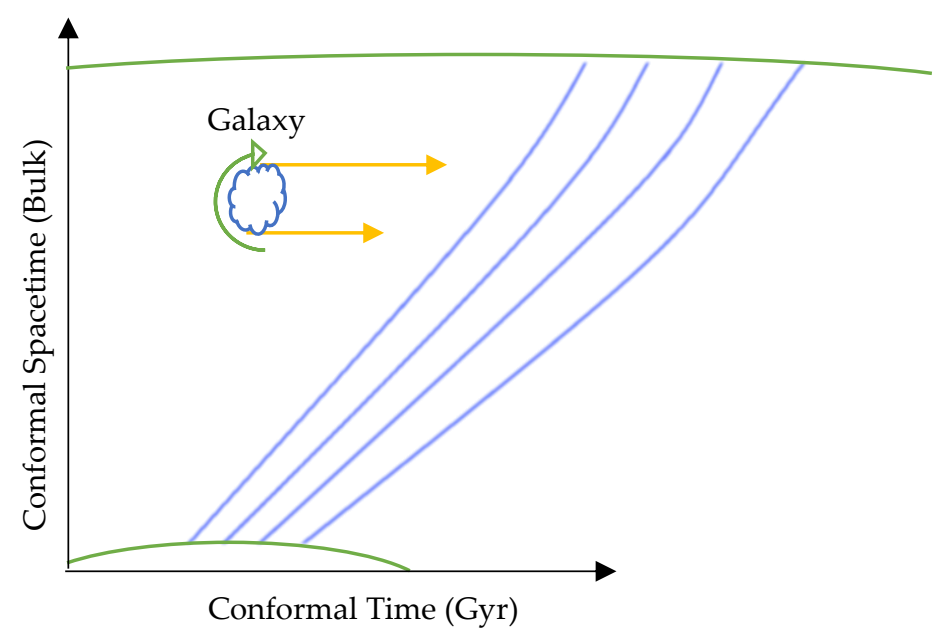

(a)

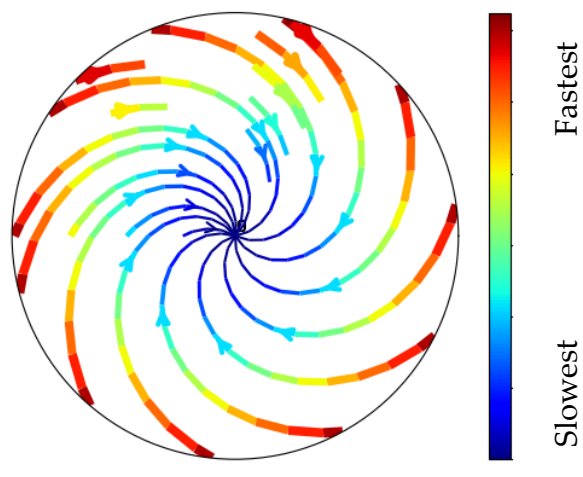

Spiral galaxy simulation

(b)

Figure 3. (a) External fields exerted on a galaxy due to the spacetime conformal curvature evolution. Green curves represent the curvature of spacetime worldlines. Blue curves represent the simulated spacetime continuum. (b) Simulation of spiral galaxy rotation. Blue represents the slowest tangential speeds and red represents the fastest speeds.

The simulation shows that the tangential speeds of the outer parts of the spiral galaxy are rotating faster in comparison with the rotational speeds of the inner parts, which resembles observations of galaxy rotation except the simulation used an ideal fluid.

\section{Conclusions and Future Works}

In this study, interaction field equations are derived in which the curvature of the background or the 4D conformal bulk evolves over the conformal time based on the PL18 recent release which has preferred a positively curved early Universe with a confidence level higher than 99\%. Throughout this bulk, 4D relativistic cloud-worlds flow and spin.

Owing to the curved background, the findings of galaxy formation showed that the core of the galaxy undergoes a forced vortex formation with a central event horizon leading to opposite traversable wormholes that are spatially shrinking through evolving in the conformal time. It revealed that the galaxy and its core form at the same process, while the gas clouds outside the core can form the spiral arms owning to the fast-rotating core that induces the frame-dragging. The formation of the galaxy and its core at the same process can explain the formation of supermassive compact galaxy cores with a mass of $\sim 10^{9} \mathrm{M} \odot$ at just $6 \%$ of the current Universe age and could solve the black hole hierarchy problem. Orbiting a wormhole could explain the observation of the G2 cloud which only faced the drag effects. In addition, the observation of the superluminal motion in the x-ray jet of M87 could be travelling through these traversable wormholes. Further, this scenario can explain the fast orbital speed of outer stars due to travelling in curved 4D conformal bulk. The derived field equations should be applied to atoms and electron clouds as that with galaxies, which can be investigated in future, where a brief is presented in Appendix 1.

Conflicts of Interest: The author declares no conflict of interest. 


\section{Appendix 1.}

In this section, the action in Equation (3) is expanded to investigate the interaction of the electron field with the electromagnetic fields under the influence of vacuum energy's field strength that in turn is dependent on the induced curvature by the presence of the conformal gravitational fields. It could be possible to expand the action using a similar procedure for an electron field or cloud which is confined in the boundary of the cloudworld as follows

$$
S=\int_{B}\left[\frac{-\mathcal{F}_{\lambda \rho} \mathcal{F}^{\lambda \rho}}{4 \mu_{0}}\right] \sqrt{-\tilde{g}} \int_{C}\left[\frac{R_{\mu \nu} g^{\mu \nu}}{\mathcal{R}_{\mu \nu} \tilde{g}^{\mu \nu}}\right] \sqrt{-g} \int_{Q}\left[\frac{D}{H}+\frac{F_{\lambda \rho} F^{\lambda \rho}}{\hat{\mathcal{F}}_{\lambda \rho} \hat{\mathcal{F}}^{\lambda \rho}}\right] \sqrt{-q} d^{4} \rho d^{4} \sigma d^{4} \alpha
$$

where $\widehat{\mathcal{F}}_{\lambda \rho} \hat{\mathcal{F}}^{\lambda \rho}$ is vacuum energy's field strength that accounts for the contributions of gravitational fields from the bulk field strength, $\mathcal{F}_{\lambda \rho} \mathcal{F}^{\lambda \rho}$, and the stress-energy from the embedded cloud-worlds. $F_{\lambda \rho} F^{\lambda \rho}$ is the applied electromagnetic field while $H$ represents the trace of the energy-momentum tensor of the vacuum energy, which could be expressed as the Klein-Gordon field as $H=H_{\mu \nu} g^{\mu \nu}=\left(\hbar^{2} / m\right)\left(g_{\mu \lambda} g_{\mu \rho}+g_{\mu \rho} g_{\mu \lambda}-g_{\mu \nu} g_{\lambda \rho}\right) \partial_{\lambda} \varphi^{*} \partial_{\rho} \varphi-$ $g_{\mu \nu} \varphi^{*} \varphi m c^{2} . D$ is the trace of the energy-momentum tensor of the quantum field and $q$ is the determinant of the field. The variation of the action gives

$$
\delta S=\int_{B}\left[\mathcal{T}_{\mu \nu}\right] \sqrt{-\tilde{g}} \int_{C}\left[\frac{G_{\mu \nu}+\mathcal{K}_{\mu \nu}}{\mathcal{R}}\right] \sqrt{-g} \int_{Q}\left[\begin{array}{l}
\frac{\delta D}{H}-\frac{D \delta H}{H^{2}}-\frac{q_{\mu \nu} \delta q^{\mu \nu}}{2 H} D+\frac{\delta F_{\lambda \rho} F^{\lambda \rho}}{\hat{\mathcal{F}}_{\lambda \rho} \hat{\mathcal{F}}^{\lambda \rho}}- \\
\frac{\delta \hat{\mathcal{F}}_{\lambda \rho} \hat{\mathcal{F}}^{\lambda \rho}}{\left(\hat{\mathcal{F}}_{\lambda \rho} \hat{\mathcal{F}}^{\lambda \rho}\right)^{2}} F_{\lambda \rho} F^{\lambda \rho}-\frac{q_{\mu \nu} \delta q^{\mu \nu}}{2 \hat{\mathcal{F}}_{\lambda \rho} \hat{\mathcal{F}}^{\lambda \rho}} F_{\lambda \rho} F^{\lambda \rho}
\end{array}\right] \sqrt{-q} d^{4} \rho d^{4} \sigma d^{4} \alpha
$$

By applying the principle of stationary action:

$$
\frac{\delta D}{H}-\frac{D}{H \ln H}=\frac{\mathcal{T}_{\mu \nu}}{\hat{\mathcal{T}}_{\mu \nu}}+\frac{F_{\mu \nu} F^{\mu \nu}}{\hat{\mathcal{T}}_{\mu \nu} \ln \hat{\mathcal{T}}_{\mu \nu}}
$$

where $\hat{\mathcal{T}}_{\mu \nu}$ is the electromagnetic stress-energy tensor of the vacuum energy's field strength based on the contributions of gravitational fields from the bulk and embedded cloud-worlds. $\mathcal{J}_{\mu \nu}$ is the applied electromagnetic stress-energy tensor of field strength $F_{\mu \nu}$. By utilizing Equations (1), the field equations can be simplified to

$$
\delta D-\frac{D}{\ln H}=\frac{8 \pi g_{x}}{c^{4}}\left(\mathcal{T}_{\mu \nu}+\frac{F_{\mu \nu} F^{\mu \nu}}{\ln \widehat{\mathcal{T}}_{\mu \nu}}\right)
$$

where $g_{x}$ is the gravity of the cloud-world as a function of the distance from its centre. Equations (4) predict a gravitational influence on the quantum field.

\section{References}

[1] Randall L and Sundrum R 1999 An alternative to compactification Phys. Rev. Lett. 83 4690-3

[2] Gogberashvili M 1999 Four Dimensionality in Non-compact Kaluza-Klein Model Mod. Phys. Lett. A 14 2025-31

[3] Randall L and Sundrum R 1999 Large Mass Hierarchy from a Small Extra Dimension Phys. Rev. Lett. 833370

[4] Di Valentino E, Melchiorri A and Silk J 2020 Planck evidence for a closed Universe and a possible crisis for cosmology Nat. Astron. 4 196-203

[5] Handley W 2021 Curvature tension: Evidence for a closed universe Phys. Rev. D 103 L041301

[6] Burkert A, Schartmann M, Alig C, Gillessen S, Genzel R, Fritz T K and Eisenhauer F 2012 Physics of the galactic center cloud G2, on its way toward the supermassive black hole Astrophys. J. 75058

[7] Becerra-Vergara E A, Argüelles C R, Krut A, Rueda J A and Ruffini R 2021 Hinting a dark matter nature of Sgr A* via the S- 
stars Mon. Not. R. Astron. Soc. Lett. 505 L64-8

[8] Landau L D 1986 Theory of Elasticity (Elsevier)

[9] Kozameh C, Newman E, gravitation K T-G relativity and and 1985 undefined 1985 Conformal Einstein spaces Springer

[10] S. M. Carroll 2003 Spacetime and Geometry: An Introduction to General Relativity

[11] Straub W O 2006 Simple Derivation of the Weyl Conformal Tensor

[12] Dyer E and Hinterbichler K 2009 Boundary terms, variational principles, and higher derivative modified gravity Phys. Rev. D - Part. Fields, Gravit. Cosmol. 79

[13] Brown J D and York J W 1993 Microcanonical functional integral for the gravitational field Phys. Rev. D 47 1420-31

[14] Rugh S E and Zinkernagel H 2000 The Quantum Vacuum and the Cosmological Constant Problem Stud. Hist. Philos. Sci. Part B - Stud. Hist. Philos. Mod. Phys. 33 663-705

[15] Gómez-Valent A 2017 VACUUM ENERGY IN QUANTUM FIELD THEORY AND COSMOLOGY

[16] Snios B, Nulsen P E J, Kraft R P, Cheung C C, Meyer E T, Forman W R, Jones C and Murray S S 2019 Detection of Superluminal Motion in the X-Ray Jet of M87

[17] Reid S, Podolefsky H and Pual A 2013 Fluid Pressure and Flow, PhET Interactive Simulations. 